Original Paper http://ajol.info/index.php/ijbcs http://indexmedicus.afro.who.int

\title{
Effet de la charge hydraulique appliquée sur le fonctionnement d'un marais artificiel à drainage vertical planté avec Panicum maximum traitant des eaux domestiques
}

\author{
Jean-Marie Pétémanagnan OUATTARA* et Lacina COULIBALY \\ Laboratoire d'Environnement et de Biologie Aquatique, UFR-Sciences et Gestion de l'Environnement, \\ Université Nangui Abrogoua, 02 BP 801 Abidjan 02, Côte d'Tvoire. \\ *Auteur correspondant ; E-mail: jm_petemanagnan@yahoo.fr ; Tel : (00225) 58050928/01642039
}

\section{RESUME}

Les marais artificiels constituent une alternative moins coûteuse pour traiter efficacement les eaux usées dans les pays en développement. Cependant, la charge hydraulique appliquée peut affecter leur efficacité de traitement. Ainsi, dans cette étude, l'effet de la charge hydraulique sur le fonctionnement d'un marais artificiel à drainage vertical planté avec Panicum maximum (Jacq.) traitant des eaux usées domestiques a été étudié. Pour ce faire, deux charges hydrauliques d'eau usée domestique $(32,14$ 1/j et 42,85 1/j) ont été utilisées. Le fonctionnement du marais artificiel a été négativement affecté par l'augmentation de la charge hydraulique. Cette augmentation de la charge hydraulique a amplifié le colmatage des réacteurs et a engendré la réduction de la biomasse végétale produite ainsi que celles des rendements épuratoires. Toutefois, les rendements obtenus avec 32,14 1/j d'eau usée appliquée sont relativement plus élevés (MES $=89,9 \pm 5,6 \%$, DCO $=89,3 \pm 5 \%$, $\mathrm{NH}_{4}{ }^{+}=80,6 \pm 6,3 \%$ et PT $=60 \pm 15,6 \%$ ). Seuls les filtrats obtenus avec la charge hydraulique de $32,141 / \mathrm{j}$ ont des concentrations de polluants qui respectent les valeurs limites indiquées dans la réglementation des rejets d'eaux usées établie en Côte d'Ivoire. Cette charge hydraulique est bien indiquée pour le fonctionnement optimal du marais artificiel planté avec P. maximum.

(C) 2019 International Formulae Group. All rights reserved

Mots clés : Charge hydraulique, marais artificiel, eau usée domestique, Panicum maximum.

\section{Effect of hydraulic load upon the operation of pilot-scale vertical flow constructed wetland planted with Panicum maximum or domestic wastewater treatment}

\begin{abstract}
ABSTRAT
Constructed wetlands are an inexpensive option for wastewater treatment in developing countries. However, the hydraulic load applied can impact their treatment efficiency. Thus, in this study, the effect of hydraulic load on the efficiency of vertical-flow constructed wetlands planted with Panicum maximum treating ran wastewater was studied. To do this, two hydraulic loads of domestic wastewater $\left(32.141 . \mathrm{d}^{-1}\right.$ and $\left.42.851 . \mathrm{d}^{-1}\right)$ were used. Constructed wetland efficiency was negatively affected by the increase of the hydraulic load. This increase of the applied hydraulic load amplified bed's clogging and led to the reduction of the plant biomass produced as well as those of bed's performance. However, pollutant removals obtained with $32.141 . \mathrm{d}^{-1}$ are
\end{abstract}


relatively higher (TSS $=89.9 \pm 5.6 \%, \mathrm{COD}=89.3 \pm 5 \%, \mathrm{NH}_{4}{ }^{+}=80.6 \pm 6.3 \%$ and $\left.\mathrm{PT}=60 \pm 15.6 \%\right)$. Only the filtrates obtained with the hydraulic load of $32.141 . \mathrm{d}^{-1}$ have pollutant concentrations that comply with the limit values indicated in the regulation of wastewater discharges established in Côte d'Ivoire. This hydraulic load is therefore well indicated for the optimal functioning of the constructed wetland planted with P. maximum.

(c) 2019 International Formulae Group. All rights reserved

Keywords: Hydraulic load, constructed wetland, domestic wastewater, Panicum maximum.

\section{INTRODUCTION}

Les eaux usées non traitées ou partiellement traitées génèrent de nombreux effets négatifs sur les milieux récepteurs tels que l'eutrophisation ou la transmission de germes pathogènes (Atinkpahoun et al., 2018). Cette situation affecte durablement la biodiversité de ces milieux et, le cas échéant, leur utilisation. Il est donc reconnu que la réduction des polluants des eaux usées est indispensable pour préserver la bonne qualité des milieux récepteurs (WWAP, 2017 ; Togbé et al., 2019). Cependant, les techniques intensives employées pour le traitement des eaux usées dans les stations de grande taille ne sont pas toujours adaptées à cause des coûts d'exploitation et de maintenance élevés et du besoin de main d'œuvre qualifiée pour opérer ces procédés. Par conséquent, le développement de nouvelles techniques devient une priorité. Ainsi, plusieurs types de bioréacteurs extensifs tels que les filtres plantés ou marais artificiels ont été développés pour répondre aux besoins du traitement des eaux usées dans les pays en développement (Coulibaly et al., 2008, Ouattara et al., 2008) et de ceux des communes rurales et décentralisées dans les pays développés (Molle et al., 2008 ; Morvannou et al., 2015).

L'épuration des eaux usées par marais artificiel présente de nombreux avantages. Il s'agit du coût d'investissement et d'exploitation relativement peu onéreux, de la faible production de boues, la facilité de maintenance et de la bonne intégration paysagère, tout en présentant un faible impact environnemental (Brix and Arias, 2005). Toutefois, la difficulté des marais artificiels réside dans le fait qu'ils sont des écosystèmes complexes nécessitant la maîtrise de plusieurs disciplines, à savoir la physiologie végétale, la chimie et l'hydraulique pour optimiser les mécanismes et équilibres qui les gouvernent (Molle, 2012 ; Vymazal, 2014). Les espèces de plantes du genre Phragmites, Typha et Cyperus, largement utilisées dans les marais artificiels dans les pays tempérés et tropicaux, sont parfois considérées comme des plantes envahissantes (Kulmatiski et al., 2010). Par conséquent, l'utilisation de plantes à intérêts économiques dans les marais artificiels leur donnerait une valeur ajoutée en leur permettant de générer des revenus pouvant assurer leur entretien et garantir leur bon fonctionnement à long terme (Coulibaly et al., 2008; Ouattara et al., 2008). Cependant, les plantes à utiliser devront être capables de résister aux conditions de fonctionnement des marais artificiels et présenter une biomasse abondante et une croissance rapide (Molle et al., 2006 ; Vymazal, 2007 ; Ngoutaneparé et al., 2012).

Concernant la charge polluante (charge organique ou minérale), elle revêt un caractère indispensable pour appréhender les conditions de fonctionnement optimal d'un marais artificiel (Molle et al., 2004; Truu et al., 2009). En effet, une charge organique ou minérale trop faible causerait un stress hydrique et minérale des plantes; ce qui nuirait aux rendements épuratoire du système (Molle et al., 2006 ; Kengne et al., 2014). Par contre, une charge organique trop élevée entraine un apport important de particules dans le substrat des réacteurs qui pourrait induire un colmatage rapide du système (Tao et al., 2006). Toutefois, contrairement au système classique de traitement des eaux usées, le procédé de marais artificiels nécessite de grandes surfaces pour son implémentation en grandeur nature (Ilyas and Masih, 2017). Par conséquent l'épuration de grands volumes d'eaux usées dans les marais 
artificiels constituerait une situation palliative. Les travaux de Ouattara et al. (2008) avec une charge hydraulique de $50 \mathrm{~L} / \mathrm{j}$ appliquée sur un marais artificiel planté avec Panicum maximum ont fourni des résultats satisfaisants de l'ordre de $74 \%, 86,5 \%, 85,5 \%$ et $91,4 \%$ de réduction, respectivement pour le phosphate, l'ammonium, les matières en suspensions (MES) et la demande chimique en oxygène (DCO). Ainsi, l'optimisation des conditions opératoires de Ouattara et al. (2008) notamment, celles relatives à la charge hydraulique appliquée permettrait d'accroitre le volume d'eau traitée par le marais artificiel développé.

L'objectif de ce travail est d'étudier l'effet de la charge hydraulique appliquée sur la croissance des plantes, le comportement hydraulique des réacteurs et les rendements épuratoires d'un marais artificiel à drainage vertical planté avec Panicum maximum pour épurer des eaux résiduaires urbaines.

\section{MATERIEL ET METHODES Dispositif expérimental}

Le dispositif sur lequel l'étude a été réalisée est un dispositif expérimental (Figure 1) situé à l'Université Nangui Abrogoua (Abidjan, Côte d'Ivoire). Il est constitué de bacs rectangulaires (longueur $\mathrm{x}$ largeur $\mathrm{x}$ hauteur $=1,75 \mathrm{~m} \times 0,75 \mathrm{~m} \times 0,45 \mathrm{~m}$ ) réalisés en briques de ciment remplis du bas vers le haut de $10 \mathrm{~cm}$ de gravier $(15 / 25 \mathrm{~mm})$ et de $30 \mathrm{~cm}$ de sable blanc de construction. Le sable et le gravier sont séparés par un géotextile. Chaque bac (réacteur) a une pente de $10 \%$ dirigée vers l'aval et équipé d'un embout de sortie pour évacuer les eaux traitées. Quatre bacs sont plantés de jeunes tiges de $P$. maximum (10 tiges $/ \mathrm{m}^{2}$ ) prélevées sur des plantes adultes et deux bacs non plantés sont pris comme témoins. Pour étudier l'effet de la charge hydraulique appliquée sur le fonctionnement $\mathrm{du}$ marais artificiel, deux charges hydrauliques ont été employées. Sur deux (2) bacs plantés et un témoin, 751 d'eau usée correspondant à un débit de $32,14 \mathrm{l} / \mathrm{j}$, soit la charge hydraulique de $2,4 \mathrm{~cm} \cdot \mathrm{j}^{-1}$, ont été apportés à chaque alimentation entre $15 \mathrm{~h}$ et $16 \mathrm{~h}$ et ce, trois (3) fois par semaine (lundi, mercredi et vendredi). Les autres bacs (deux
(2) plantés et un (1) non planté) ont été alimentés, dans les mêmes conditions, avec 1001 d'eau usée par application, soit un débit de $42,85 \mathrm{l} / \mathrm{j}$ qui correspond à la charge hydraulique de $3,2 \mathrm{~cm} . j^{-1}$. L'expérience a duré six mois.

\section{Croissance et biomasse végétale produite}

La croissance des plantes a été suivie par la mesure hebdomadaire de la longueur de cinq tiges (une par angle (4) et au centre (1) $\mathrm{du}$ réacteur) à l'aide d'un ruban gradué en millimètre. Au début du stade de montaison (production de graines) correspondant à la fin du cycle de croissance des tiges (deux mois environ), les plantes ont été fauchées. Trois (3) opérations de fauchage ont été effectuées pendant l'essai de traitement. Les biomasses végétales produites à l'état frais et sec (après séchage à l'étuve à $70{ }^{\circ} \mathrm{C}$ pendant $72 \mathrm{~h}$ ) ont été déterminées par pesée sur une balance (SARTORIUS EB150FEG-I) de précision 5 $10^{-3} \mathrm{~kg}$. La production journalière de biomasse végétale a été calculée suivant la relation (1).

$$
\begin{aligned}
& \mathrm{P}= \frac{\mathrm{BV}}{\Delta t \times \mathrm{S}} \\
& \mathrm{P}=\text { Production journalière }\left(\mathrm{t} / \mathrm{j} \cdot \mathrm{ha}^{-1}\right) ; \\
& \mathrm{BV}=\text { Biomasse fraîche ou sèche }(\mathrm{t}) ; \\
& \Delta t=\text { Durée entre deux fauchages }(\mathrm{j}) ; \\
& \mathrm{S}=\text { Surface du réacteur }(\mathrm{ha}) .
\end{aligned}
$$

\section{Caractérisation hydraulique des réacteurs}

Cette caractérisation a été faite en déterminant le débit d'infiltration de l'eau usée dans les réacteurs, le temps de séjour hydraulique et le pourcentage de colmatage des lits. Le débit d'infiltration de l'eau usée a été obtenu à partir de la mesure du temps d'infiltration de la lame d'eau appliquée sur chaque réacteur pendant les jours d'alimentation $\mathrm{du}$ marais artificiel. Cette mesure a consisté à relever, à l'aide d'un chronomètre, le temps que met la lame d'eau usée appliquée pour s'infiltrer et disparaître totalement de la surface du réacteur. Le débit d'infiltration est le rapport du volume d'eau usée appliquée et le temps d'infiltration correspondant (Equation 2).

$$
\mathrm{Q}=\frac{\mathrm{V}_{\mathrm{e}}}{t}
$$


$\mathrm{Q}=$ Débit d'infiltration ( $\mathrm{ml} / \mathrm{min})$;

$\mathrm{V}_{\mathrm{e}}=$ Volume d'eau usée appliquée (ml);

$t=$ Temps d'infiltration de la lame d'eau (min).

Echantillonnage d'eau, analyses physicochimiques et rendements épuratoires

Durant les essais de traitement, des échantillons d'eau ont été prélevés dans des flacons en polyéthylène de 0,51 , une fois par semaine (tous les mercredis), à l'entrée (eau usée brute) et à la sortie (eau usée traitée) de chaque réacteur, puis conservés à $4{ }^{\circ} \mathrm{C}$ pendant $24 \mathrm{~h}$ avant les analyses au laboratoire. Sur chaque échantillon d'eau prélevée le $\mathrm{pH}$, les matières en suspension (MES), la demande chimique en oxygène (DCO), l'ammonium $\left(\mathrm{NH}_{4}{ }^{+}\right)$et le phosphore total (PT) ont été déterminés selon les méthodes AFNOR (AFNOR, 2005). Pour chaque charge hydraulique appliquée, les charges massiques apportée dans les réacteurs, celles traitées et celles restituées ont été calculées.
Les rendements épuratoires, à partir desquels la performance épuratoire du marais artificiel a été évaluée, ont été calculés selon l'équation 3.

$$
=\frac{\begin{array}{c}
\text { Rendement Epuratoire (\%) } \\
\text { Charge entrée }- \text { Charge sortie }
\end{array}}{\text { Charge entrée }} \times 100
$$

Charge entrée $=$ Concentration de l'eau usée brute $\mathrm{x}$ charge hydraulique ;

Charge sortie $=$ Concentration de l'eau traitée $\mathrm{x}$ charge hydraulique.

\section{Analyses statistiques}

Une analyse de variance (ANOVA) à un facteur a été utilisée pour tester la variation de la croissance des plantes, de la biomasse végétale produite et des rendements épuratoires entre les différents réacteurs. Elle a été suivie du test HSD de Tukey pour la comparaison des valeurs moyennes de ces paramètres entre les réacteurs pris deux à deux. Elle a été réalisée à l'aide du logiciel STATISTICA 7.1.
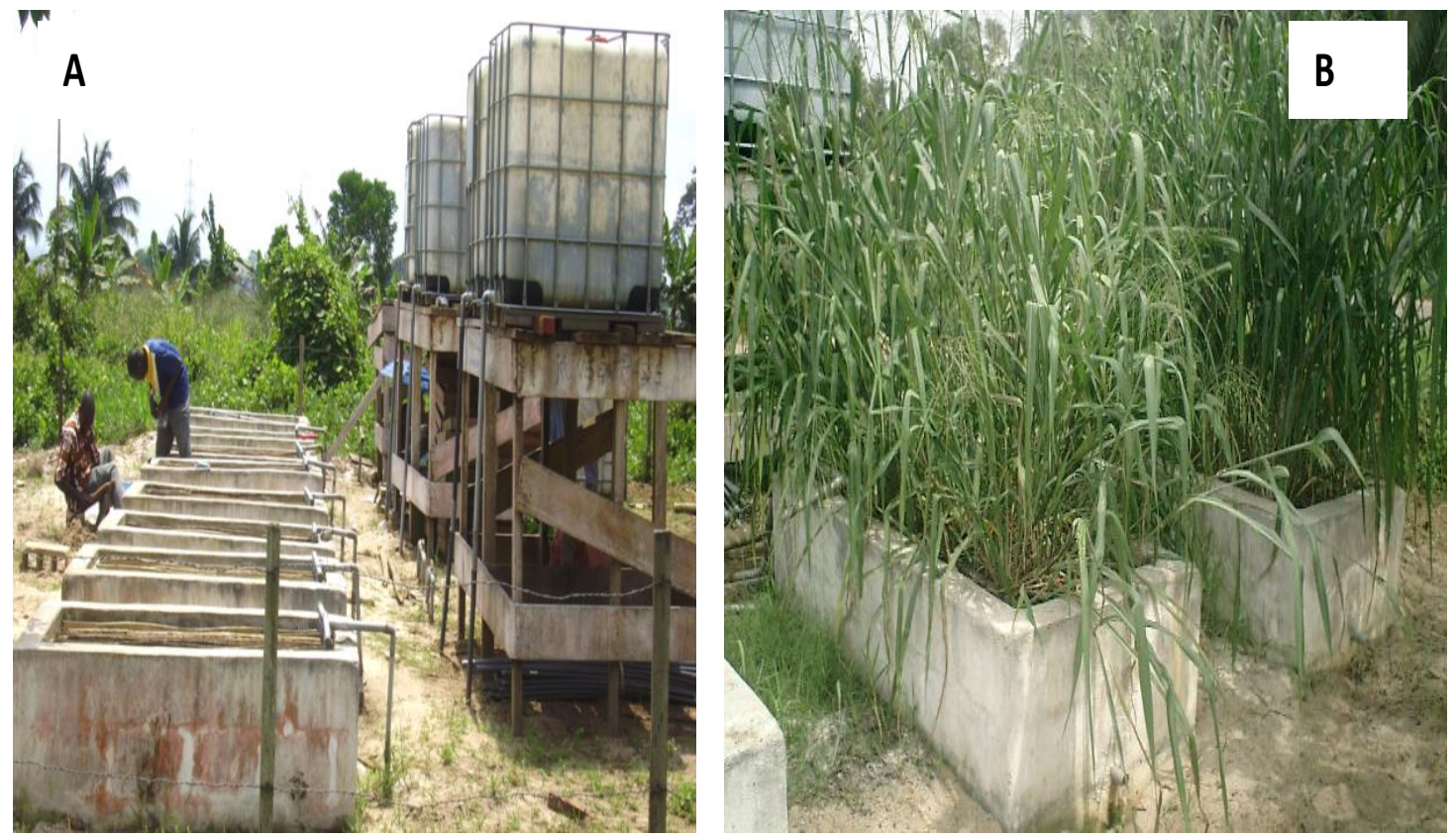

Figure 1 : Vue du dispositif expérimental (A) et des réacteurs planté avec P. maximum (B). 


\section{RESULTATS}

\section{Croissance des plantes et biomasses végétales produites}

La Figure 2 présente la croissance de $P$. maximum pendant les trois périodes de repousse durant l'essai de traitement. Les plantes ont atteint, au premier, second et troisième fauchage, des longueurs maximales respectives de $129,9,117,8$ et $102 \mathrm{~cm}$ sur le réacteur alimenté avec $32,14 \mathrm{l} / \mathrm{j}$ contre 124 , 102 et $95,6 \mathrm{~cm}$ sur celui alimenté avec 42,85 $1 / \mathrm{j}$. La longueur moyenne des plantes sur le réacteur alimenté avec 32,14 1/j (116,6 \pm 14 $\mathrm{cm}$ ) ne diffère pas statistiquement (test HSD de Tukey: $\mathrm{p}>0,05)$ de celle du réacteur alimenté avec 42,85 1/j (107,2 $\pm 14,9 \mathrm{~cm})$. Les deux types de réacteurs plantés (RP $(32,14)$ et RP $(42,85)$ ) ont produit des biomasses végétales fraîche et sèche qui s'élèvent, du premier au troisième fauchage, respectivement de 68,8 à 117,8 t.ha $^{-1}$ et de 10,1 à 22,8 t.ha $^{-1}$ (Figure 3). Dans l'ensemble, les biomasses végétales produites par le réacteur alimenté avec 32,14 $1 / \mathrm{j}$ d'eau usée (biomasse fraîche = 73,8 à 117,8 t.ha $^{-1}$ et biomasse sèche $=13,4$ à 22,8 t.ha $^{-1}$ ) sont supérieures à celles produites par le réacteur alimenté avec 42,85 $1 / \mathrm{j}$ (biomasse fraîche $=68,8$ à 91,4 t.ha $^{-1}$ et biomasse sèche $=10,1$ à 17,9 t.ha $\left.{ }^{-1}\right)$. La production journalière oscille entre 1,2 et 2,1 $t / j$.ha ${ }^{-1}$ pour la biomasse fraîche et entre 0,2 et $0,4 \mathrm{t} / \mathrm{j} \cdot \mathrm{ha}^{-1}$ pour la biomasse sèche dans le réacteur RP $(32,14)$. En revanche, dans celui alimenté avec $42,851 / \mathrm{j}$, elle est comprise entre 1,4 et $1,9 \mathrm{t} / \mathrm{j} \cdot \mathrm{ha}^{-1}$ pour la biomasse fraîche et entre 0,3 et $0,4 \mathrm{t} / \mathrm{j} \cdot \mathrm{ha}^{-1}$ pour la biomasse sèche. Toutefois, les valeurs obtenues ne diffèrent pas significativement d'un réacteur à l'autre (test HSD de Tukey : $\mathrm{p}>0,05$ ).

\section{Hydraulique des réacteurs}

Les profils de débit pour les charges hydrauliques établies sont semblables et présentent plusieurs phases marquées par des pics (Figure 4). Pendant ces phases, le débit d'infiltration diminue avec la durée du fonctionnement des réacteurs. Concernant les réacteurs alimentés avec 32,14 1/j (Figure 4A), les profils de débit présentent trois phases distinctes. Dans le réacteur planté, le débit d'infiltration diminue de $647,4 \mathrm{ml} / \mathrm{min}$ au jour 1 à $167,2 \mathrm{ml} / \mathrm{min}$ au jour 63 pendant la première phase, de $619,3 \mathrm{ml} / \mathrm{min}$ au jour 67 à $175,6 \mathrm{ml} / \mathrm{min}$ au jour 130 pendant la seconde phase et de $580,7 \mathrm{ml} / \mathrm{min}$ au jour 133 à 232,3 $\mathrm{ml} / \mathrm{min}$ au jour 183 pendant la troisième phase. Dans celui non planté, le débit d'infiltration décroît de 573,9 à $144,8 \mathrm{ml} / \mathrm{min}$, de 323,2 à $151,5 \mathrm{ml} / \mathrm{min}$ et de 323,5 à 162,4 $\mathrm{ml} / \mathrm{min}$, respectivement pendant les première (jours 1 à 42), deuxième (jours 44 à 93) et troisième (jours 95 à 183) phases. Quant aux réacteurs alimentés avec 42,85 1/j (Figure 4B), les profils du débit d'infiltration montrent quatre phases. Les valeurs enregistrées dans le réacteur planté baissent de 495,6 à 183,8 $\mathrm{ml} / \mathrm{min}$ du jour 1 au jour 42 (première phase), de 393,8 à 182,9 ml/min du jour 44 au jour 93 (seconde phase), de 367,4 à $184,5 \mathrm{ml} / \mathrm{min}$ du jour 95 au jour 133 (troisième phase) et de 362,1 à 227,2 $\mathrm{ml} / \mathrm{min}$ du jour 135 au jour 183 (quatrième phase). Dans le témoin, elles passent de 369,5 ml/min (jour 1) à 149,4 $\mathrm{ml} / \mathrm{min}$ (jour 35), de 265,2 ml/min (jour 37) à $140 \mathrm{ml} / \mathrm{min}$ (jour 77), de $221,5 \mathrm{ml} / \mathrm{min}$ (jour 79) à $142,3 \mathrm{ml} / \mathrm{min}$ (jour 137) et de 218,2 $\mathrm{ml} /$ min (jour 140) à $135,5 \mathrm{ml} / \mathrm{min}$ (jour 180), respectivement aux première, deuxième, troisième et quatrième phases.

Quelle que soit le volume d'eau usée appliqué $(32,14 \mathrm{~L} / \mathrm{j}$ ou $42,85 \mathrm{~L} / \mathrm{j})$, les débits d'infiltration de l'eau usée dans les réacteurs plantés sont significativement plus élevés (test HSD de Tukey : $\mathrm{p}<0,05)$ que ceux dans les réacteurs non plantés. En considérant les réacteurs plantés (Figure 4C), les valeurs de débit obtenues dans le réacteur alimenté avec $32,14 \mathrm{~L} / \mathrm{j}(410,7 \pm 59,6 \mathrm{~mL} / \mathrm{min})$ restent significativement supérieures (test HSD de Tukey: $\mathrm{p}<0,05)$ à celles relevées dans celui alimenté avec $42,85 \mathrm{~L} / \mathrm{j} \quad(259,9 \pm 56,2$ $\mathrm{mL} / \mathrm{min}$ ).

\section{Performances épuratoires des réacteurs pH}

L'évolution du $\mathrm{pH}$ de l'eau usée brute (EB) et des filtrats des réacteurs alimentés avec $32,14 \mathrm{l} / \mathrm{j}$ et $42,85 \mathrm{l} / \mathrm{j}$ est indiquée par la Figure 5. Le $\mathrm{pH}$ de l'eau usée utilisée est compris entre 7,9 et 9,3 , avec une valeur 
moyenne de 8,4 $\pm 0,4$ tandis que dans les filtrats des réacteurs alimentés avec $32,14 \mathrm{l} / \mathrm{j}$ (Figure 5A), les valeurs oscillent entre 6,4 et 8 pour le réacteur planté $(\operatorname{RP}(32,14))$ et entre 6,3 et 8,2 pour celui non planté (RNP $(32,14))$. Concernant les réacteurs alimentés avec $42,85 \mathrm{l} / \mathrm{j}$ d'eau usée (Figure 5B), les valeurs de $\mathrm{pH}$ varient entre 7,1 et 8,3 et entre 6,1 et 8,3 , respectivement dans les filtrats des réacteurs plantés et non plantés. Dans l'ensemble, le $\mathrm{pH}$ des filtrats des différents réacteurs est plus faible que celui de l'eau usée. Cependant, les valeurs ne diffèrent pas significativement entre l'eau usée brute et les filtrats des différents réacteurs (test HSD de Tukey: $p>0,05)$. S'agissant des filtrats des réacteurs, les valeurs de $\mathrm{pH}$ sont relativement plus faibles dans les réacteurs alimentés avec $32,141 / \mathrm{j}(7,1$ à 7,6$)$ que dans ceux alimentés avec $42,851 / j$ ( $(7,6$ à 7,9$)$.

\section{Matières en suspension}

Pendant l'essai de traitement, la charge massique des matières en suspension (MES) diminue significativement (test HSD de Tukey: $\mathrm{p}<0,05)$ dans les filtrats des différents réacteurs par rapport à l'eau usée brute (Figure 6). A l'entrée des réacteurs, les charges massiques journalières sont en moyenne de $29,6 \pm 9,7 \mathrm{~g}$ et de $39,5 \pm 12,9 \mathrm{~g}$ respectivement pour les réacteurs alimentés avec $32,14 \mathrm{l} / \mathrm{j}$ et ceux alimentés avec $42,85 \mathrm{l} / \mathrm{j}$. A la sortie des réacteurs alimentés avec 32,14 $1 / \mathrm{j}$, la charge massique journalière passe à 2,97 $\pm 0,2 \mathrm{~g}$ dans le réacteur planté $(\operatorname{RP}(32,14))$ et à $2,21 \pm 1,7 \mathrm{~g}$ dans celui non planté (RNP $(32,14))$. Pour les réacteurs alimentés avec $42,85 \mathrm{l} / \mathrm{j}$, la charge massique journalière moyenne est de $3,21 \pm 1,5 \mathrm{~g}$ à la sortie du réacteur planté $(\mathrm{RP}(42,85))$ et de $1,93 \pm 1,00$ $\mathrm{g}$ à la sortie de celui non planté (RNP $(42,85))$. Entre les deux (2) charges hydrauliques appliquées, la différence de charge massique journalière à la sortie des réacteurs n'est pas significative (test HSD de Tukey : $\mathrm{p}>0,05)$. Les rendements épuratoires moyens obtenus sont de $89,97 \pm 5,60 \%$ et de $92,53 \pm 5,96 \%$ respectivement pour les réacteurs plantés et non planté alimentés avec $32,141 / \mathrm{j}$, et de $91,87 \pm 4,42 \%$ et de $95,11 \pm$ $2,43 \%$ respectivement pour les réacteurs plantés et non planté alimentés avec 42,85 1/j. Ils ne diffèrent pas significativement ( $\mathrm{p}>$ $0,05)$ d'un réacteur à l'autre.

\section{Demande chimique en oxygène}

La Figure 7 illustre l'évolution de la charge massique journalière de la Demande chimique en oxygène (DCO) à l'entrée (eau usée brute $(\mathrm{EB})$ ) et à la sortie (filtrats) des réacteurs alimentés avec $32,14 \mathrm{l} / \mathrm{j}$ et $42,85 \mathrm{l} / \mathrm{j}$ d'eau usée. Durant l'essai de traitement, les charges massiques de la DCO à la sortie des réacteurs restent nettement inférieures à celles à l'entrée des réacteurs (Figures 7A et 7B). Les charges massiques moyennes apportées dans les réacteurs sont de $49,32 \pm 10,17 \mathrm{~g}$ et $65,75 \pm 8,9 \mathrm{~g}$ respectivement pour les réacteurs alimentés avec $32,14 \mathrm{l} / \mathrm{j}$ et pour ceux alimentés avec $42,85 \quad 1 / j$. Les valeurs moyennes rejetées dans les filtrats sont de $5,29 \pm 0,95 \mathrm{~g}$ et de $20,18 \pm 6,55 \mathrm{~g}$, et $20,66 \pm$ $2,31 \mathrm{~g}$ et de $28,48 \pm 6,81 \mathrm{~g}$, respectivement à la sortie des réacteurs planté et non planté alimentés avec 32,14 1/j (RP $(32,14)$ et RNP $(32,14)$ ) et ceux alimentés avec 42,85 1/j (RP $(42,85)$ et RNP $(42,85))$. Les rendements épuratoires fournis par les réacteurs alimentés avec $42,85 \mathrm{l} / \mathrm{j}$ sont significativement plus élevés que ceux fournis par les réacteurs alimentés avec 32,14 1/j (test HSD de Tukey: $\mathrm{p}<0,05)$. Les rendements épuratoires moyens correspondants sont de $89,27 \pm 5,04 \%$ pour le réacteur planté alimenté avec 32,14 1/j (RP $(32,14))$, de $59,08 \pm 15,64 \%$ pour son homologue non planté (RNP $(32,14)$ ), de $68,57 \pm 5,47 \%$ pour le réacteur planté alimenté avec 42,85 1/j (RP $(42,85)$ ) et de $56,68 \pm 8,27 \%$ pour celui non planté alimenté avec $42,85 \mathrm{l} / \mathrm{j}$ (RNP $(42,85))$.

\section{Ammonium}

Les profils de la charge massique de l'ammonium $\left(\mathrm{NH}_{4}{ }^{+}\right)$dans l'eau usée brute (EB) (entrée) et dans les filtrats (sortie) des réacteurs plantés et non plantés alimentés avec $32,14 \mathrm{l} / \mathrm{j}$ et $42,85 \mathrm{l} / \mathrm{j}$ d'eau usée sont présentés par la Figure 8. Les valeurs obtenues dans les filtrats des différents réacteurs sont plus faibles que celles contenues dans l'eau usée appliquée. Celles-ci sont plus petites dans les réacteurs plantés que dans les réacteurs non plantés (Figures 8A et 8B). En comparaison 
avec les charges massiques à la sortie des réacteurs alimentés avec $42,85 \mathrm{l} / \mathrm{j}$, celles des réacteurs alimentés avec $32,14 \mathrm{l} / \mathrm{j}$ présentent des valeurs plus faibles. A l'entrée des réacteurs, les charges massiques sont de 4,23 $\pm 0,89 \mathrm{~g}$ et de $5,64 \pm 1,10 \mathrm{~g}$ respectivement pour les charges hydrauliques de $32,14 \mathrm{l} / \mathrm{j}$ et de 42,85 $\mathrm{l} / \mathrm{j}$ tandis qu'à la sortie des réacteurs, elles sont de $0,82 \pm 0,2 \mathrm{~g}$ pour RP $(32,14)$, de $2,38 \pm 0,8$ g pour $\operatorname{RNP}(32,14)$, de $1,96 \pm 0,82$ $\mathrm{g}$ pour RP $(42,85)$ et de $3,34 \pm 1,18 \mathrm{~g}$ pour $\operatorname{RNP}(42,85)$.

L'abattement de $\mathrm{NH}_{4}{ }^{+}$obtenu dans le réacteur planté alimenté avec 32,14 1/j d'eau usée (RP $(32,14)=80,61 \pm 6,3 \%)$ est significativement plus élevé (test HSD de Tukey : $\mathrm{p}<0,05$ ) que celui enregistré dans le réacteur planté alimenté avec 42,85 1/j $(\operatorname{RP}(42,85)=65,24 \pm$ $12 \%)$.

\section{Phosphore total}

La Figure 9 présente les variations de la charge massique du phosphore total (PT) dans l'eau usée brute (EB) et dans les filtrats des réacteurs du marais artificiel. Dans l'ensemble, on observe une baisse de la charge polluante de ce paramètre à la sortie comparativement à celle à l'entrée des réacteurs. Cette réduction de la charge polluante de PT est plus significative à la sortie des réacteurs plantés comparativement à ceux non plantés quelle que soit la charge hydraulique appliquée (test HSD de Tukey : $p$ $<0,05)$. Les rendements épuratoires moyens fournis par les réacteurs se présentent dans l'ordre suivant : $\operatorname{RP}(32,14)=56,7 \pm 15,6 \%>$ $\operatorname{RP}(42,85)=41,4 \pm 26,4 \%>\operatorname{RNP}(32,14)=$ $30,00 \pm 18,2 \%>\operatorname{RNP}(42,85)=26,92 \pm 11 \%$.

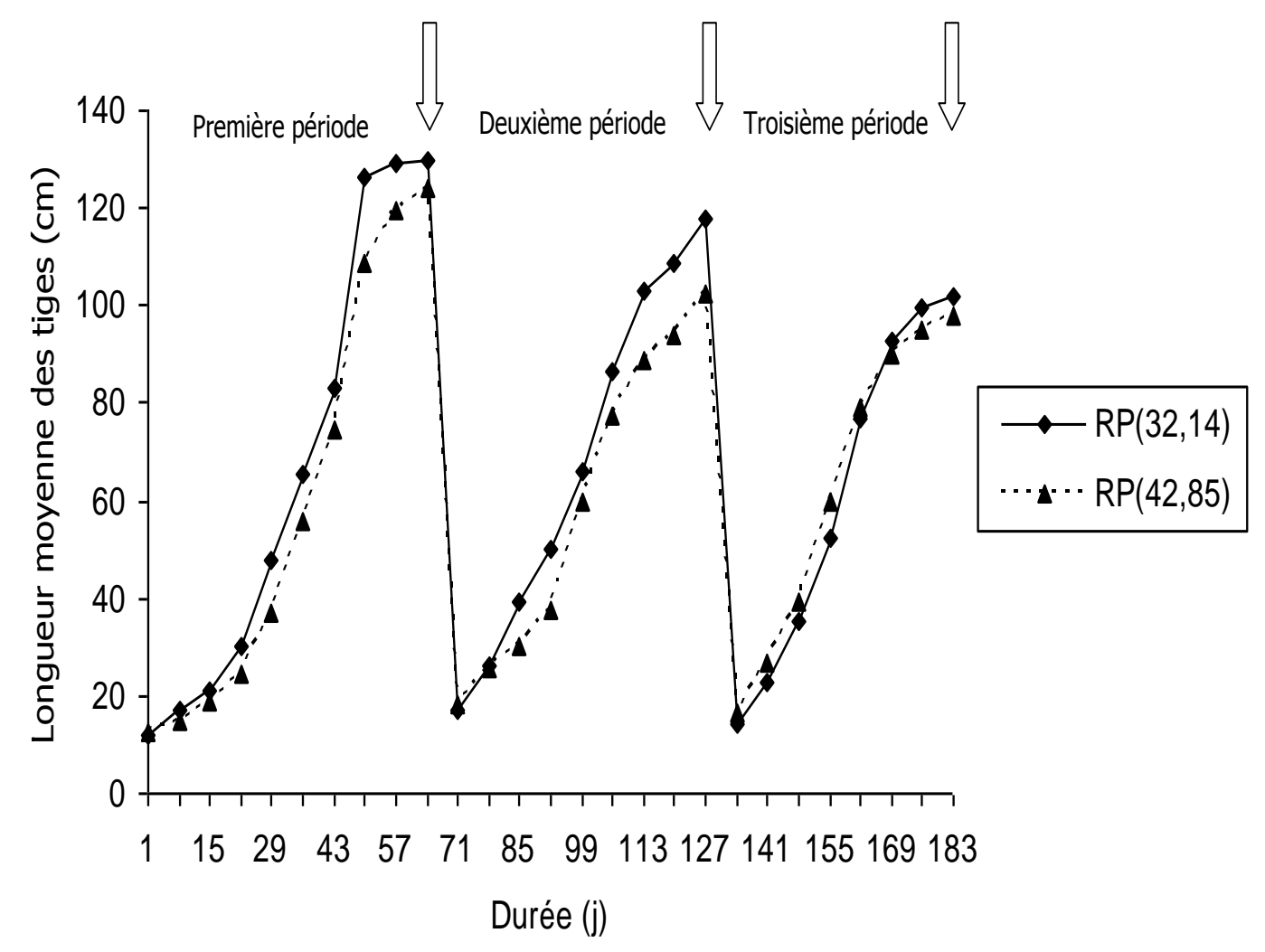

Figure 2 : Profils de croissance des tiges de P. maximum pendant trois cycles de croissance; les flèches indiquent les moments de fauchage des plantes ; RP $(32,14)=$ réacteur planté alimenté avec $32,141 / \mathrm{j} ; \mathrm{RP}(42,85)=$ réacteur planté alimenté avec 42,85 1/j. 

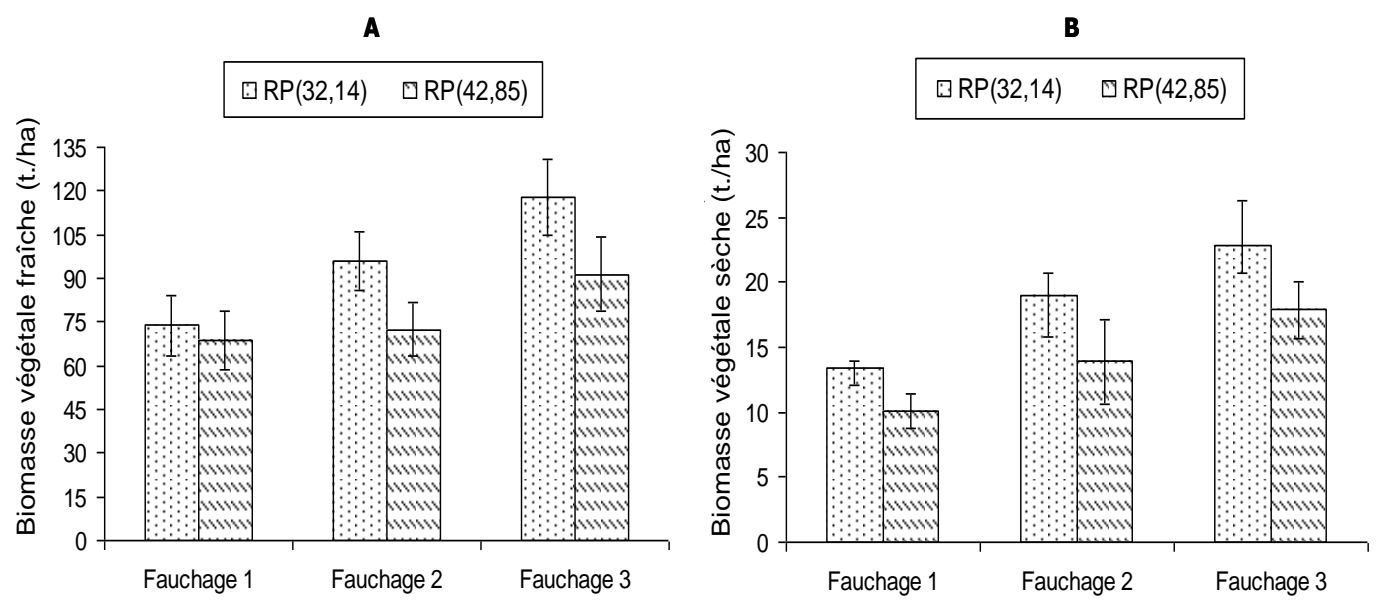

Figure 3 : Variation des biomasses végétales fraîche $(\mathrm{A})$ et sèche $(\mathrm{B})$ de $P$. maximum produites pendant trois périodes de fauchage ; $\operatorname{RP}(32,14)=$ réacteur planté alimenté avec $32,141 / \mathrm{j} ; \mathrm{RP}$ $(42,85)=$ réacteur planté alimenté avec $42,85 \mathrm{l} / \mathrm{j}$.
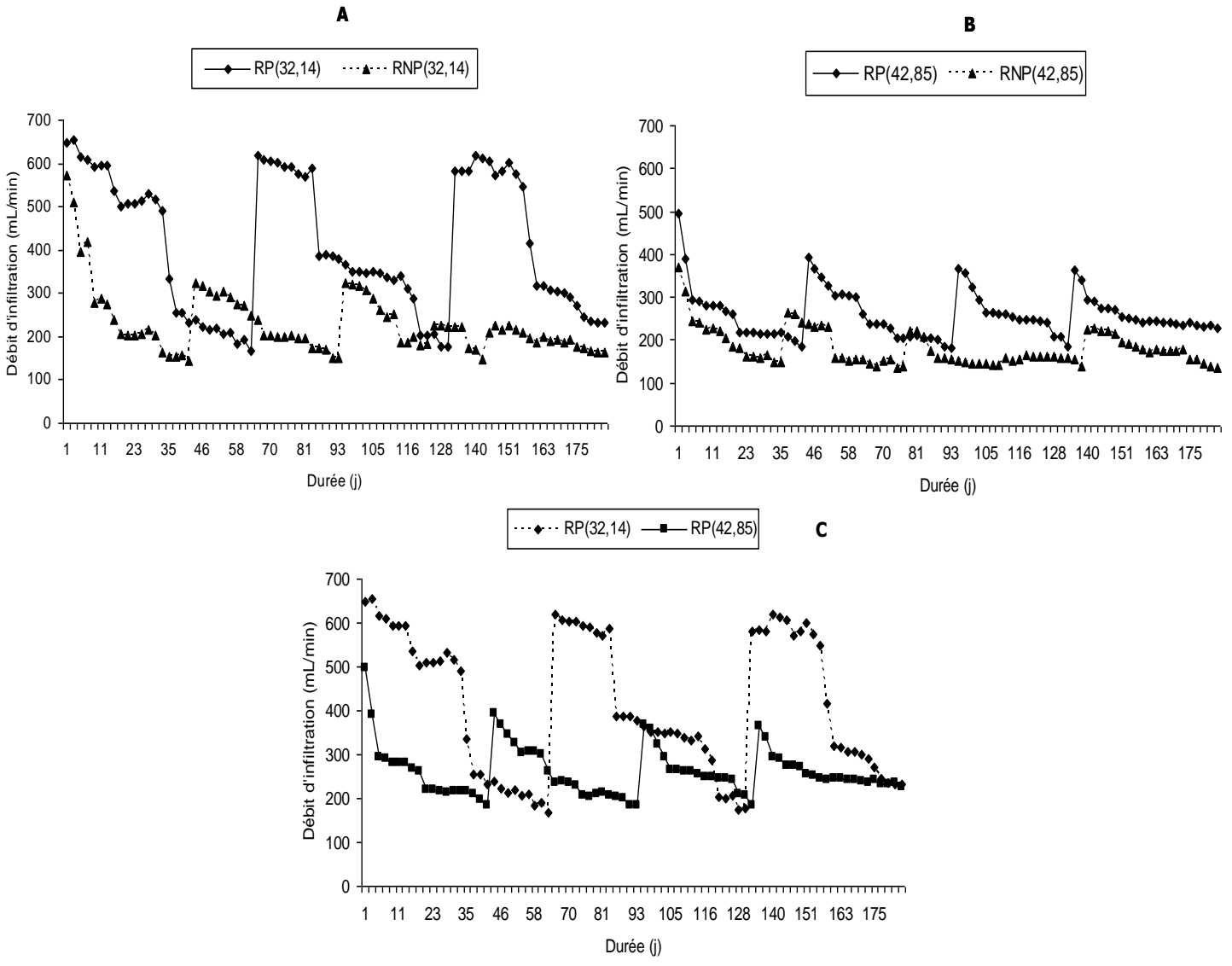

Figure 4 : Évolution du débit d'infiltration de l'eau usée dans les réacteurs du marais artificiel ; RP $(32,14)=$ réacteur planté alimenté avec $32,141 / \mathrm{j} ; \mathrm{RNP}(32,14)=$ réacteur non planté alimenté avec $32,141 / \mathrm{j} ; \operatorname{RP}(42,85)=$ réacteur planté alimenté avec 42,85 1/j ; RNP $(42,85)=$ réacteur non planté alimenté avec $42,85 \mathrm{l} / \mathrm{j}$. 

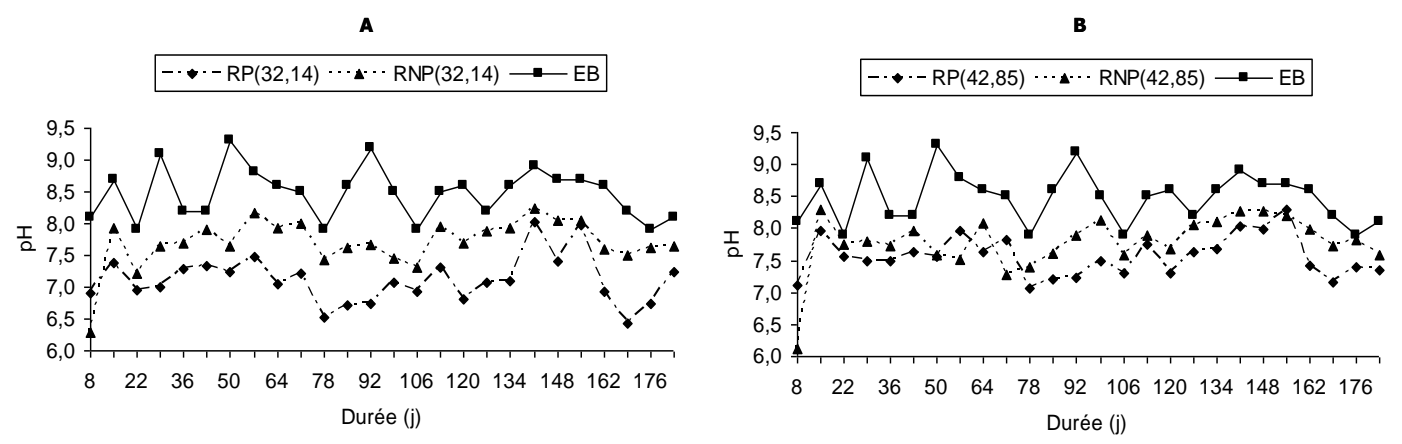

$\cdots \cdot \mathrm{RP}(32,14) \rightarrow-\mathrm{RP}(42,85) \quad \mathrm{C}$

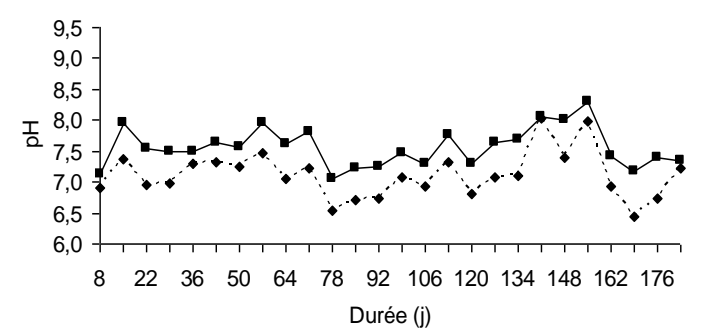

Figure 5 : Évolution du pH de l'eau usée (EB) et des filtrats des réacteurs du marais artificiel ; RP $(32,14)=$ réacteur planté alimenté avec $32,141 / \mathrm{j} ; \mathrm{RNP}(32,14)=$ réacteur non planté alimenté avec $32,141 / \mathrm{j} ; \operatorname{RP}(42,85)=$ réacteur planté alimenté avec 42,85 1/j ; RNP $(42,85)=$ réacteur non planté alimenté avec $42,85 \mathrm{l} / \mathrm{j}$.
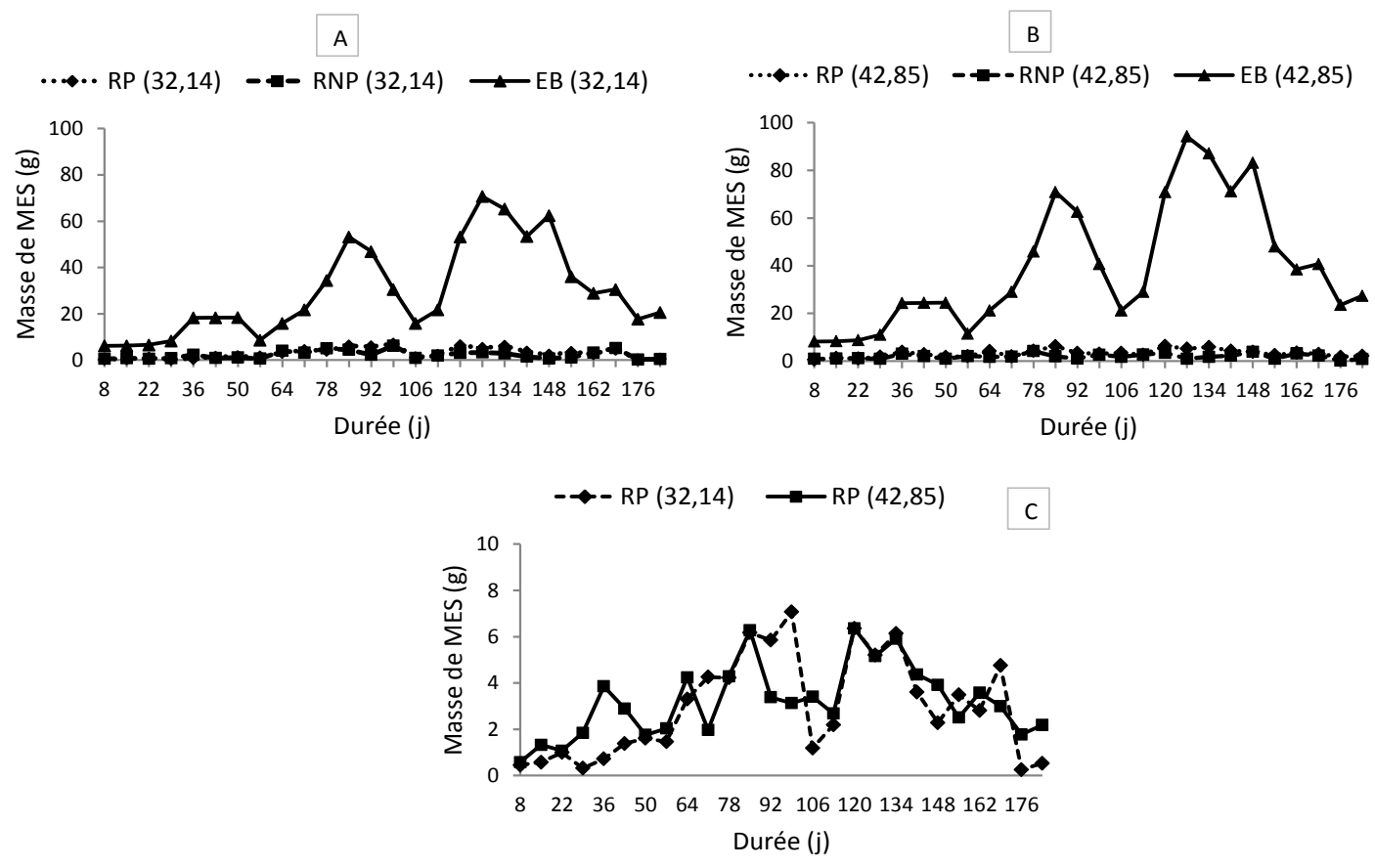

Figure 6 : Évolution de la charge massique des MES dans l'eau brute (EB) et dans les filtrats des réacteurs ; RP $(32,14)=$ réacteur planté alimenté avec $32,141 / j ; \operatorname{RNP}(32,14)=$ réacteur non planté alimenté avec $32,141 / \mathrm{j} ; \operatorname{RP}(42,85)=$ réacteur planté alimenté avec 42,85 1/j ; RNP $(42,85)=$ réacteur non planté alimenté avec $42,85 \mathrm{l} / \mathrm{j}$. 

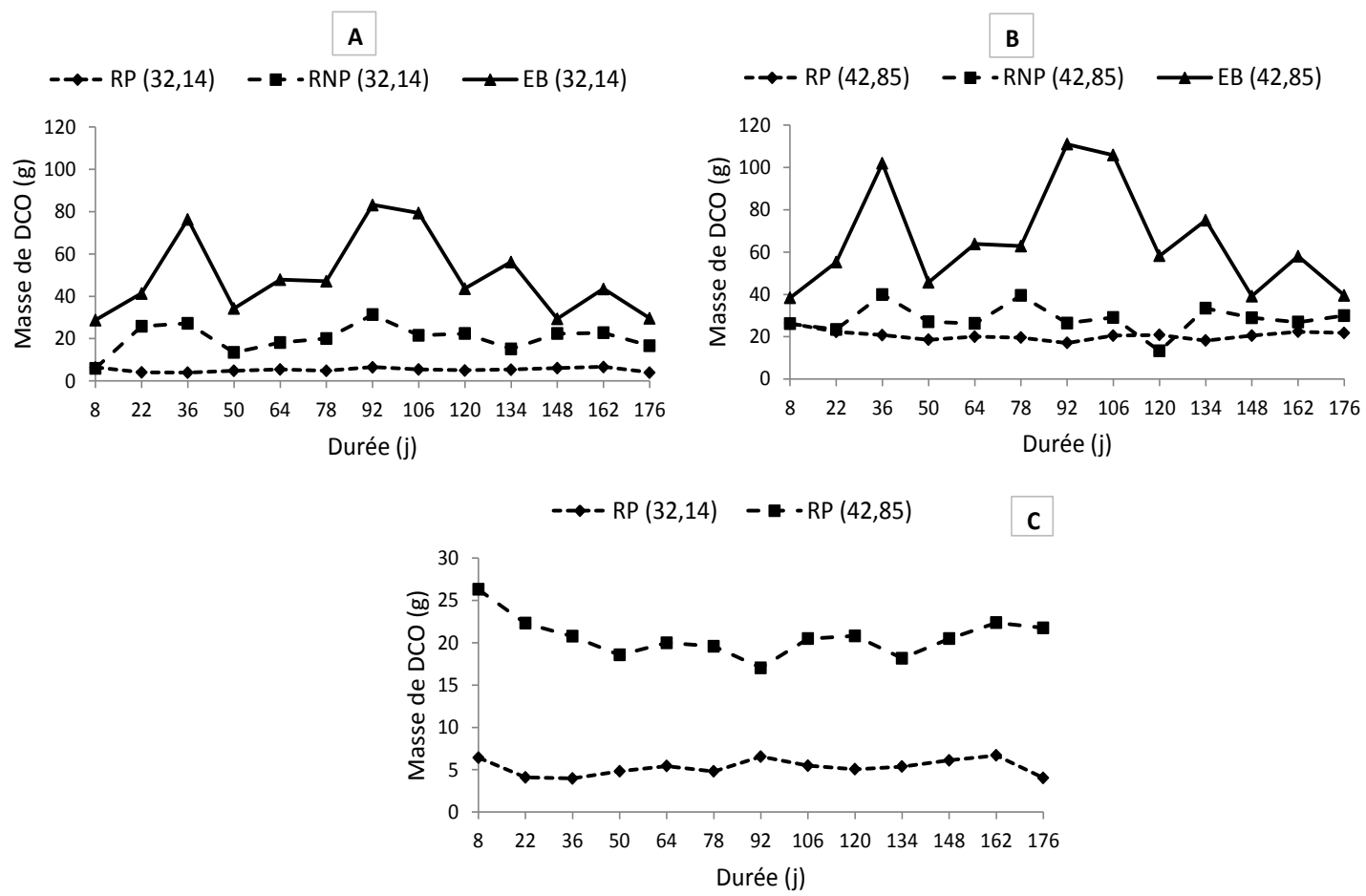

Figure 7 : Évolution de la charge massique de la DCO dans l'eau usée et dans les filtrats des réacteurs ; RP $(32,14)=$ réacteur planté alimenté avec $32,141 / \mathrm{j} ; \operatorname{RNP}(32,14)=$ réacteur non planté alimenté avec 32,14 1/j; RP $(42,85)$ = réacteur planté alimenté avec 42,85 1/j; RNP $(42,85)=$ réacteur non planté alimenté avec $42,851 / \mathrm{j}$.
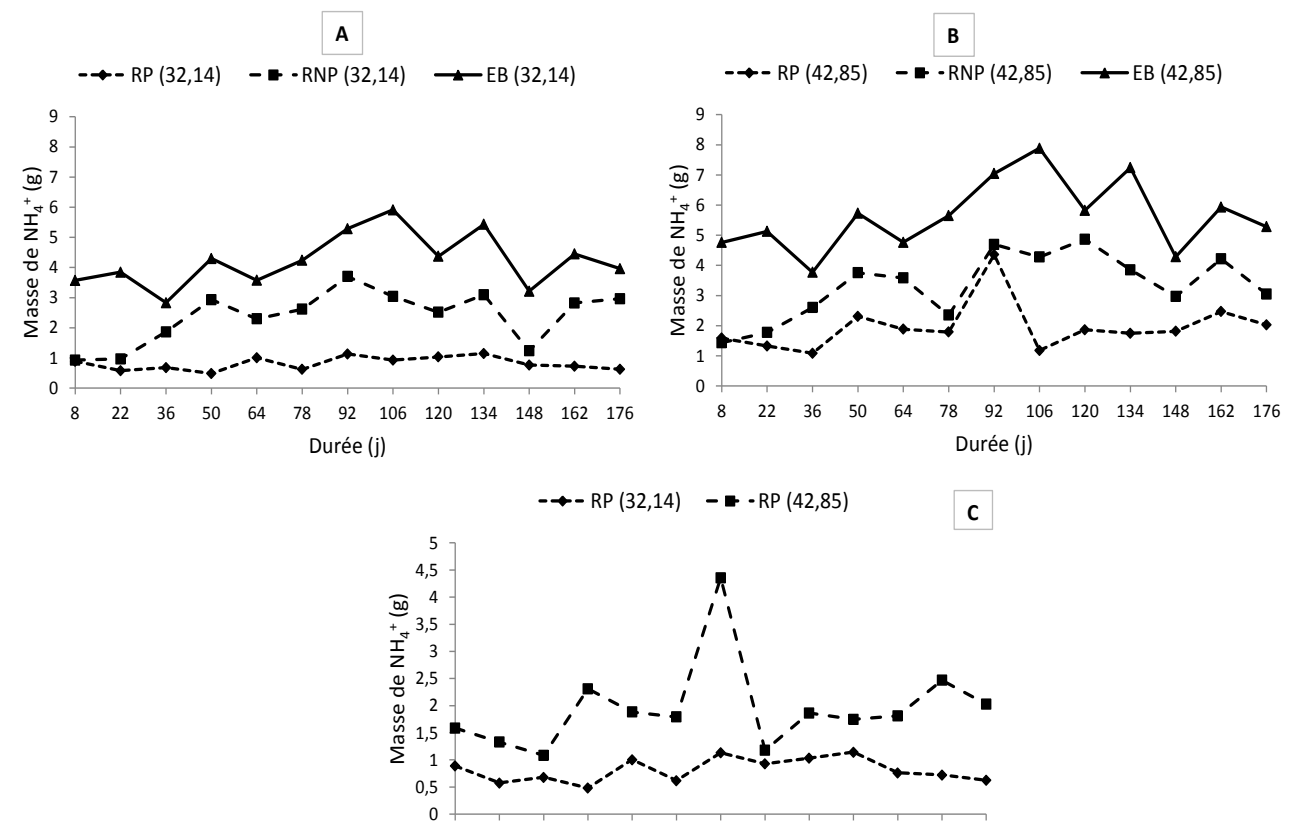

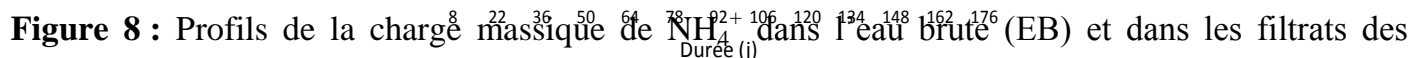
réacteurs durant l'essai de traitement; $\mathrm{RP}(32,14) \stackrel{\text { (1) }}{=}$ réacteur planté alimenté avec $32,14 \mathrm{l} / \mathrm{j} ; \mathrm{RNP}$ $(32,14)$ = réacteur non planté alimenté avec $32,14 \mathrm{l} / \mathrm{j} ; \mathrm{RP}(42,85)$ = réacteur planté alimenté avec $42,85 \mathrm{l} / \mathrm{j} ; \operatorname{RNP}(42,85)=$ réacteur non planté alimenté avec $42,85 \mathrm{l} / \mathrm{j}$. 

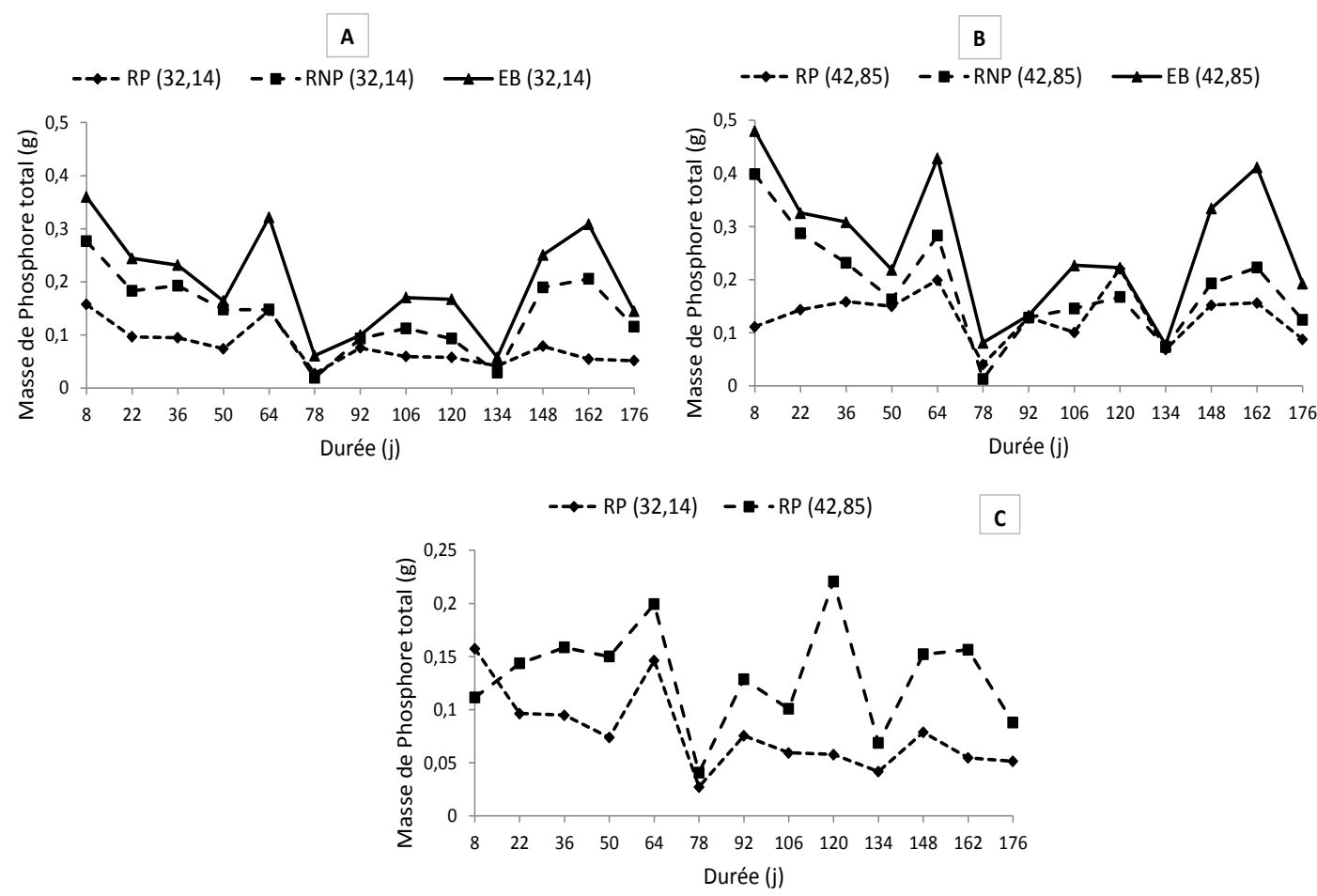

Figure 9: Variation de la charge massique de PT dans l'eau brute (EB) et dans les filtrats des réacteurs ; RP $(32,14)=$ réacteur planté alimenté avec $32,141 / j ; \operatorname{RNP}(32,14)=$ réacteur non planté alimenté avec $32,14 \mathrm{l} / \mathrm{j} ; \mathrm{RP}(42,85)=$ réacteur planté alimenté avec 42,85 1/j ; RNP $(42,85)=$ réacteur non planté alimenté avec 42,85 1/j.

\section{DISCUSSION}

Les résultats de cette étude ont montré une incidence de la charge hydraulique appliquée sur le fonctionnement du marais artificiel. En effet, l'augmentation du débit d'alimentation de $32,14 \quad 1 / \mathrm{j}$ à $42,85 \mathrm{l} / \mathrm{j}$ a entraîné une diminution de la croissance des plantes, de la biomasse végétale produite, de l'hydraulique des réacteurs et des rendements épuratoires. S'agissant de la croissance des plantes et de la biomasse végétale produite, les deux débits appliqués n'influent pas significativement sur les valeurs obtenues. Dans les deux cas, $P$. maximum s'est bien acclimatée aux milieux de culture, vraisemblablement à cause de sa nature physiologique. En fait, cette plante est fortement hydrophile et sa croissance nécessite des quantités importantes de matières organiques et nutritives (Muir and Jank, 2004 ; Klomjek, 2016). Toutefois, les feuilles des plantes du réacteur alimenté à un débit de 42,85 1/j d'eau usée avaient une coloration plus verte que celles des plantes du réacteur alimenté avec $32,14 \mathrm{l} / \mathrm{j}$. Cette observation pourrait résulter selon Zahui et al. (2018), d'un enrichissement important du substrat du réacteur RP $(42,85)$ en nutriments et l'absorption de ceux-ci par les plantes. Cependant, on observe une diminution de la biomasse végétale produite et du diamètre des souches des plantes lorsque le débit d'eau usée augmente. Un résultat similaire a été obtenu par Durand (2007) qui l'ont attribué à la surcharge (asphyxie) du substrat des réacteurs lorsque la charge hydraulique augmentait. Seules, les plantes du réacteur alimenté avec $32,14 \mathrm{l} / \mathrm{j}$ d'eau usée produisent une biomasse végétale sèche $\left(18,4 \pm 4,7 \mathrm{t}_{\mathrm{h}} \mathrm{ha}^{-1}\right)$ conforme aux estimations de Séfiétou et al. (2005).

Le suivi de l'hydraulique des réacteurs a mis en exergue une baisse du débit d'infiltration de l'eau usée dans les réacteurs au cours de l'essai de traitement. La diminution du débit d'infiltration est plus 
marquée lorsque la charge hydraulique augmente. Les réacteurs alimentés ave 32,14 $1 / \mathrm{j}$ d'eau usée présentent des débits d'infiltration significativement plus élevés que ceux des réacteurs alimentés au débit d'eau usée de 42,85 1/j. La baisse du débit d'infiltration dans les réacteurs pourrait s'expliquer par la réduction de l'espace porale du substrat de ceux-ci par les particules solubles ou en suspension contenues dans l'eau usée (Liénard et al., 2001 ; Cooper et al., 2004) et par la croissance de la flore microbienne (Wu and Huang, 2000). L'augmentation de la charge hydraulique appliquée entraîne un apport important de particules (charge polluante) dans le substrat des réacteurs qui réduit considérablement la vitesse d'infiltration de l'eau (Prochaska et al., 2007 ; Bastviken et al., 2009). A cet effet, Molle et al. (2006) ont rapporté que les caractéristiques du substrat des réacteurs (structure, porosité et humidité) se dégradent avec l'augmentation de la charge hydraulique et la durée de fonctionnement des marais artificiels. En outre, l'augmentation de la charge hydraulique accroît l'humidité du substrat et réduit la conductivité hydraulique des réacteurs (Molle et al., 2006). Toutefois, le fonctionnement hydraulique du marais artificiel est assuré pendant deux (2) mois lorsqu'on applique 32,14 1/j d'eau usée.

Dans l'ensemble, les réacteurs alimentés avec $32,14 \mathrm{l} / \mathrm{j}$ (soit $2,4 \quad \mathrm{~cm} \cdot \mathrm{j}^{-1}$ ) réduisent significativement la charge polluante de l'eau usée par rapport à ceux alimentés avec 42,85 1/j (soit $3,2 \quad \mathrm{~cm} \cdot \mathrm{j}^{-1}$ ). Concernant le pH, l'on a observé une tendance à la basicité des filtrats lorsque le débit d'alimentation augmente de 32,14 $1 / \mathrm{j}$ à $42,85 \mathrm{l} / \mathrm{j}$. Ce résultat pourrait s'expliquer par une diminution de l'aération du substrat des réacteurs due à l'augmentation de la charge polluante apportée; ce qui réduirait la nitrification et l'oxydation de la matière organique qui devraient acidifier l'eau usée. Des résultats similaires ont été mentionnés par certains auteurs qui les ont expliqués par le même phénomène (Tchobanoglous et al., 2003 ; Bojcevska and Tonderski, 2007). Les filtrats du réacteur planté alimenté avec 32,14 $1 / \mathrm{j}$ ont un $\mathrm{pH}$ plus proche de la neutralité $(7,1)$ et peuvent intégrer les milieux aquatiques sans nuisance, car respectant les normes de rejet des eaux usées en Côte d'Ivoire pour ce paramètre (Ministère de l'Environnement, des Eaux et Forêts, 2008).

L'élimination des MES dans les différents réacteurs est très élevée, mais ne présente pas de différences significatives entre ces réacteurs. Cette situation est vraisemblablement due au fait que l'élimination des MES dans les marais artificiels est principalement assurée par des mécanismes physiques et dépend donc des caractéristiques du matériau de filtration utilisé (Diop et al., 2014 ; Abdelhakeem et al., 2016). Or, dans cette étude, les caractéristiques des réacteurs du pilote expérimental sont identiques. Toutefois, l'on note une amélioration de la réduction des MES avec l'augmentation de la charge hydraulique appliquée tout comme Chazarenc et al. (2007) l'ont observée lors de leurs travaux sur des marais artificiels. Ce résultat serait imputable à une réduction significative de la porosité du substrat des réacteurs due à l'élévation de la charge polluante avec l'augmentation du débit appliqué.

L'abattement de la DCO est négativement corrélé à l'augmentation de la charge hydraulique appliquée. Il est de 89,27 $\pm 5,04 \%$ dans le réacteur alimenté avec 32,14 $1 / \mathrm{j}$ et de $68,57 \pm 5,47 \%$ dans celui alimenté avec $42,851 / \mathrm{j}$. La différence de performance entre les deux types de réacteurs est attribuable à la fois à la réduction de l'aération des réacteurs et celle du temps de séjour hydraulique. En effet, l'augmentation du débit d'alimentation anticipe le colmatage des réacteurs par un apport important de charge polluante Chazarenc et al. (2007) qui ralentit le transfert d'oxygène par convection et par diffusion dans le substrat des réacteurs ; ce qui limite l'oxydation microbienne de la matière organique (Kayser and Kunst, 2005 ; Molle et al., 2006; Molle et al., 2008). Le débit de $32,14 \mathrm{l} / \mathrm{j}$ a permis d'obtenir une concentration moyenne de DCO des filtrats de 164,6 $\pm 29,5$ $\mathrm{mg} \mathrm{O}_{2} / 1$ qui est largement inférieure à la valeur limite $\left(300 \mathrm{mg} \mathrm{O} \mathrm{O}_{2} / \mathrm{l}\right.$ ) fixée par la réglementation de rejets d'eau usée en Côte d'Ivoire (Ministère de l'Environnement, des Eaux et Forêt, 2008).

S'agissant de $\mathrm{NH}_{4}{ }^{+}$, son faible abattement dans le réacteur alimenté avec $42,851 / \mathrm{j}(65,24 \pm 12 \%)$ par rapport à celui 
alimenté au débit de 32,14 1/j $(80,61 \pm 6,3 \%)$ pourrait s'expliquer par la limitation du transfert d'oxygène dans le réacteur alimenté avec $42,85 \mathrm{~L} / \mathrm{j}$ à cause de l'augmentation de la charge polluante apportée. Cette situation réduit alors l'activité des bactéries nitrifiantes (Molle et al., 2006 ; Bojcevska and Tonderski, 2007 ; Prochaska et al., 2007). En dépit de l'augmentation de la charge hydraulique, les rendements épuratoires de $\mathrm{NH}_{4}{ }^{+}$obtenus sont relativement élevés pour un marais artificiel à forte charge de $\mathrm{NH}_{4}^{+}$.

L'augmentation de la charge hydraulique appliquée affecte également l'élimination du phosphore total de l'eau usée. Ce résultat est probablement dû au fait que l'adsorption et la précipitation dominent les mécanismes d'élimination du phosphore dans les marais artificiels. Par conséquent, l'augmentation du débit d'alimentation provoque le lessivage du phosphore adsorbé et limite les réactions de précipitation. A ce sujet, Molle et al. (2006) ont montré que l'augmentation de la charge hydraulique entraîne une élévation de la charge massique des polluants dans les réacteurs; ce qui provoque une saturation des surfaces actives du substrat et réduit l'élimination de ces polluants par adsorption.

\section{Conclusion}

Le présent travail a permis d'étudier l'influence de la charge hydraulique appliquée sur la croissance des plantes, le comportement hydraulique des réacteurs et les rendements épuratoires d'un marais artificiel à drainage vertical planté avec Panicum maximum pour épurer des eaux résiduaires urbaines. Il ressort que l'augmentation de la charge hydraulique affecte négativement le fonctionnement du marais artificiel. Certes, les plantes de $P$. maximum se sont bien développées en produisant une biomasse végétale abondante avec les différentes charges hydrauliques appliquées, mais cette biomasse a diminué avec l'augmentation de la charge hydraulique. L'élévation de la charge hydraulique a accentué le colmatage des réacteurs. Cependant, avec la charge hydraulique de 2,4 $\mathrm{cm} . \mathrm{j}^{-1}$, soit le débit d'eau usée de $32,14 \mathrm{l} / \mathrm{j}$, le fonctionnement hydraulique des réacteurs est assuré pendant deux (2) mois. En outre, l'efficacité épuratoire du marais artificiel régresse avec l'augmentation de la charge hydraulique appliquée. Les rendements épuratoires des charges organique et nutritive obtenus avec les deux débits d'alimentation expérimentés $(32,14 \mathrm{l} / \mathrm{j}$ et $42,85 \mathrm{l} / \mathrm{j})$ sont relativement élevés. Cependant, seuls les filtrats du réacteur planté et alimenté avec $32,14 \mathrm{l} / \mathrm{j}$ ont des concentrations de polluants qui respectent les valeurs limites indiquées dans la réglementation des rejets d'eaux usées établie en Côte d'Ivoire.

\section{CONFLIT D'INTERETS}

Les auteurs déclarent qu'ils n'ont pas de conflit d'intérêts par rapport à cet article.

\section{CONTRIBUTIONS DES AUTEURS}

JMPO a assuré la conduite de l'expérience et la rédaction du manuscrit. LC a assuré la conception du pilote expérimental et la supervision des travaux.

\section{REMERCIEMENTS}

Nous remercions l'Unité de Recherche en Biotechnologie et Ingénierie Environnementale pour l'assistance technique et les suggestions.

\section{REFERENCES}

Abdelhakeem SG, Aboulroos SA, Kamel MM. 2016. Performance of a vertical subsurface flow constructed wetland under different operational conditions. $J$. $A d v$. Res., 7(5): 803-814. DOI: doi.org/10.1016/j.jare.2015.12.002

AFNOR 2005. Recueil Normes et Réglementation Environnement. Qualité de l'eau, vol. 1, $552 \mathrm{p}$.

Atinkpahoun $\mathrm{CNH}$, Soclo $\mathrm{HH}$, Pons MN, Leclerc JP. 2018. Physico-chemical characterization of domestic wastewaters in the "Vie-Nouvelle" agglomeration, Cotonou, Benin. Int. J. Biol. Chem. Sci., 12(1): $542-557 . \quad$ DOI: https://dx.doi.org/10.4314/ijbcs.v12i1.42

Bastviken SK, Weisner SEB, Thiere G., Svensson JM, Ehde PM, Tonderski KS. 2009. Effects of vegetation and hydraulic load on seasonal nitrate removal in treatment wetlands. Ecol. Eng., 35(5): 946-952.

DOI: 
Brix H, Arias AC. 2005. The use of vertical flow constructed wetlands for on-site treatment of domestic wastewater: New Danish guidelines. Ecol. Eng., 25(5): 491-500. DOI: 10.1016/j.ecoleng.2005.07.009

Bojcevska H, Tonderski K. 2007. Impact of loads, season, and plant species on the performance of a tropical constructed wetland polishing effluent from sugar factory stabilization ponds. Ecol. Eng. 29(1): $\quad 66-76 . \quad$ DOI: 10.1016/j.ecoleng.2006.07.015

Chazarenc F, Maltais-Landry G, Troesch S, Comeau Y., Brisson J. 2007. Effect of loading rate on performance of constructed wetlands treating an anaerobic supernatant. Water Sci. Technol., $\quad \mathbf{5 6}(3)$ : 23-29. DOI: 10.2166/wst.2007.500

Cooper D, Griffin P, Cooper PF. 2004. Facteurs affectant la longévité des filtres à écoulement horizontal en traitement tertiaire d'effluents domestiques. Ingénieries $N^{\circ}$ spécial, 49-56.

Coulibaly L, Kouakou J, Savané I, Gourène G. 2008. Domestic wastewater treatment with a vertical completely drained pilot scale constructed wetland planted with Amaranthus hybridus. Africa Journal of Biotechnology, 7(15): 2656-2664. DOI: https://doi.org/10.5897/AJB07.634

Diop C, Diarra MD, Sonko EHM, Tiné M, F Matty, Silva AD, Dioné IPM, Tiné A. 2014. Experimental study of slow sand filtration for the treatment of various wastewaters in tropical environment. Int. J. Biol. Chem. Sci., 8(6): 2828-2841. DOI: http://dx.doi.org/10.4314/ijbcs.v8i6.39

Durand JL. 2007. Les effets du déficit hydrique sur la plante : aspects physiologiques. Fourrages, 190: 181195.

Ilyas H, Masih I. 2017. Intensification of constructed wetlands for land area reduction: a review. Environ Sci Pollut Res Int., 24(13): 12081-12091. DOI: http://doi.org/10.1007/s11356-017-8740-z

Kayser K, Kunst S. 2005. Processes in vertical-flow reeds beds-nitrification, oxygen transfer and soil clogging. Water
Sci. Technol., 51(9): 177-184. DOI: 10.2166/wst.2005.0314

Kengne ES, Kengne IM, Nzouebet AL, Akoa A, Hung NV, Strande L. 2014. Performance of vertical flow constructed wetlands for faecal sludge drying bed leachate: Effect of hydraulic loading. Ecol. Eng., 71: 384-393. DOI: dx.doi.org/10.1016/j.ecoleng.2014.07.041

Klomjek P. 2016. Swine wastewater treatment using vertical subsurface flow constructed wetland planted with Napier grass. Sust. Environ. Res., 26(5): 217223.

DOI: https://doi.org/10.1016/j.serj.2016.03.001

Kulmatiski A, Beard K, Meyerson L. 2010. Nonnative Phragmites australis invasion into Utah wetlands. West. N Am Nat. 70(4): $\quad 541-552 . \quad$ DOI: 10.3398/064.070.0414

Liénard A, Guellaf H, Boutin C. 2001. Choise of the sand for sand filters used for secondary treatment of wastewater. Water Sci. Technol., 44(2-3): 189-196. DOI:

https://doi.org/10.2166/wst.2001.0769

Ministère de l'Environnement, des Eaux et Forêts $2008 . \quad$ Arrêté $\mathrm{N}^{\circ}$ 01164/MINEEF/CIAPOL/SDIIC du 04 Nov. 2008 portant Réglementation des Rejets et Emissions des Installations Classées pour la Protection de l'Environnement. Ministère de l'Environnement, des Eaux et Forêts/Centre Ivoirien Antipollution, Côte d'Ivoire.

Molle P. 2012. Les filtres plantés de roseaux : évolution de la recherche et tendances actuelles. Sciences Eaux et Territoires, 09 : 24-3. DOI: https://hal.archivesouvertes.fr/hal-00772874

Molle P, Liénard A, Boutin C, Merlin G, Iwema A. 2004. Traitement des eaux usées domestiques par marais artificiels : état de l'art et performances des filtres plantés de roseaux en France. Ingénieries $N^{\circ}$ spécial, 23-32.

Molle P, Liénard A, Grasmick A, Iwema A. 2006. Effect of reeds and feeding operations on hydraulic behaviour of vertical flow constructed wetlands under hydraulic overloads. Water Res., 40(3): 
606-612.

10.1016/j.watres.2005.11.026

DOI:

Molle P, Prost-Boucle S, Liénard A. 2008. Potential for total nitrogen removal by combining vertical flow and horizontal flow constructed wetlands: A full-scale experiment study. Ecol. Eng., 34(1): 2329. DOI: 10.1016/j.ecoleng.2008.05.016

Morvannou A, Forquet N, Michel S, Troesch S, Molle P. 2015. Treatment performances of French constructed wetlands: Results from a database collected over the last 30 years: Water Sci. Technol., 71(9): 1333 -1339. DOI: 10.2166/wst.2015.089

Muir JP, Jank L. 2004. Guineagrass. In Moser LE, Burson BL, Sollenberger LE. (Eds.) Warm-Season (C4) Grasses. Agron Monogr 45. ASA, CSSA, SSSA, Madison, Wi, 589-621.

Ngoutaneparé MM, Dongo K, Kengne IM, Dodané PH, Akoa A, Koné D. 2012. The economic potential of Echinochloa pyramidalis (Lam.) Hitchc \& Chase forage plant used in liquid waste treatment in Cameroon: opportunity to link sanitation to food security. Int. J. Biol. Chem. Sci., 6(1): 210-236. DOI: http://dx.doi.org/10.4314/ijbcs.v6i1.19

Ouattara PJM, Coulibaly L, Manizan P, Gourène G. 2008. Traitement des eaux résiduaires urbaines par un marais artificiel à drainage vertical planté avec Panicum maximum sous climat tropical. European Journal of Science Research, 23(1): 25-40. http://www.eurojournals.com/ejsr.htm

Prochaska CA, Zouboulis AI, Eskridgeb KM. 2007. Performance of pilot-scale vertical-flow constructed wetlands, as affected by season, substrate, hydraulic load and frequency of application of simulated urban sewage. Ecol. Eng., 31(1): 57-66. DOI: https://doi.org/10.1016/j.ecoleng.2007.05 .007

Safiétou TF, Rippstein G, Corniaux C. 2005. Les fourrages et les aliments du bétail. In Bilan de la recherche agricole et agroalimentaire au Sénégal, ISRA-ITACIRAD (ed). ISRA-ITA-CIRAD : Sénégal, 267-272.
Tao W, Hall K, Duff S. 2006. Performance evaluation and effects of hydraulic retention time and mass loading rate on treatment of woodwaste leachate in surface flow constructed wetlands. Ecol. Eng. 26, 252-265. DOI: 10.1016/j.ecoleng.2005.10.006

Tchobanoglous G, Burton FL, Stensel HD. 2003. Wastewater Engineering: Treatment and Reuse (4 ${ }^{\text {th }}$ Edn). McGraw-Hill: New York.

Togbé AMO, Kouamé KV, Yao KM, Ouattara AA, Tidou AS, Atsé BC. 2019. Évaluation de la contamination des eaux de la lagune Ebrié (Zones IV et V), Côte d'Ivoire en arsenic, plomb et cadmium: variations spatio-temporelles et risques sanitaires. Int. J. Biol. Chem. Sci., 13(2): 1162-1179.

DOI: https://dx.doi.org/10.4314/ijbcs.v13i2.45

Truu M, Juhanson J, Truu J. 2009. Microbial biomass, activity and community composition in constructed wetlands. Sci. Total Environ., 407(13): 3958-3971. DOI: $10.1016 /$ j.scitotenv.2008.11.036

Vymazal J. 2014. Constructed wetlands for treatment of industrial wastewaters: a review. Ecol. Eng., 73: 724-751. DOI: 10.1016/j.ecoleng.2014.09.034

Wu FC, Huang HT. 2000. Hydraulic resistance induced by deposition of sediment in porous medium. $J$. Hydraulic Eng., 126: 547-551. http://www.bse.ntu.edu.tw/wfc/pdf/A05.pdf

WWAP (Programme mondial pour l'évaluation des ressources en eau) 2017. Rapport mondial des Nations Unies sur la mise en valeur des ressources en eau. Les eaux usées - Une ressource inexploitée. UNESCO: Paris, 204 p.

Zahui FM, Ouattara PJM, Messou A, Coulibaly L. 2018. Nutrient removal and balance in vertical-flow constructed wetlands planted with different forage macrophytes for domestic wastewater treatment. Int. J. Dev. Res., 8(6): 2104621055.

DOI: http://www.journalijdr.com/archive/2018 06 